\title{
Passive energy balancing design for a linear actuated morphing
}

\section{wingtip structure}

\author{
Jiaying Zhang ${ }^{1}$, Chen Wang ${ }^{* 1}$, Alexander D. Shaw ${ }^{1}$, Mohammadreza \\ Amoozgar $^{2}$, and Michael I. Friswell ${ }^{1}$ \\ 1. College of Engineering, Swansea University, Swansea SA1 8EN, United Kingdom \\ 2. School of Computing and Engineering, University of Huddersfield, Huddersfield HD1 3DH, United \\ Kingdom \\ *E-mail: chen.wang@swansea.ac.uk
}

\begin{abstract}
A passive energy balancing concept for linear actuation is investigated in the current work by adopting a negative stiffness mechanism. The proposed negative stiffness mechanism uses a pre-tensioned spring to produce a passive torque and therefore to transfer the passive torque through a crankshaft for linear motion.

The proposed passive energy balancing design is supposed to be applied in a morphing wingtip, of which the shape change comes from the elastic deformation of the morphing structure. A significant amount of linear actuation force can be required to deform the structure, and therefore it is important to reduce the required force and the consumed energy by adopting the passive energy balancing design.

The kinematics of the negative stiffness mechanism is developed to satisfy the required linear motion and its geometry is then optimised to reduce the energy requirements. The performance of the optimised negative stiffness mechanism is evaluated through the net
\end{abstract}


force and the total required energy, which shows the potential of the design in the morphing wingtip application.

Keyword. negative stiffness mechanism; kinematics tailoring; energy balancing; actuator efficiency; morphing wingtip; morphing aircraft; 


\section{Introduction}

Morphing aircraft have the capability of shape-changing, and have the potential to improve aerodynamic performance under different flight conditions [1-3]. Morphing wingtips, which introduce shape changes in the wingtips, could achieve relatively-high performance improvements through moderate shape-changing. Improvement in the fuel efficiency of a business jet is obtained using camber morphing in the winglet [4]. An aero-servo-elastic design of the morphing trailing edge in the winglet is established and used to analyse the full-scale wing model [5]. A bio-inspired morphing wing, which has the variable sweep angle close to the wingtip, has shown optimal lift-to-drag ratios at different Reynolds numbers [6] and its control method is investigated with deep learning [7]. Bistable winglets, which can snap from a planar wing geometry to a folded geometry, can be achieved using composite layup design [8] or a metallic structure with polymer [9]. The change of the winglet dihedral angle can lead to the redistribution of the wing loads. A passive folding wingtip device, which has a hinge and springs between the main wing and wingtip, that allows changes to the wingtip folding angle, has been investigated for gust load alleviation [10-12].

To achieve the shape change, traditional mechanisms and smart structures are designed using ordinary actuators and or smart materials to overcome the internal resistance (e.g. structure deformation) and external loads (e.g. aerodynamic load). These integrated systems allow shape changes of the structure, and thus control its motion with acceptable accuracy. Traditional actuators, such as electromechanical devices, can be used as linear and rotary actuators, combined with mechanisms to provide a powerful tool for morphing [13]. Moreover, smart materials provide alternative ways to design actuation systems compared to the conventional actuation systems based on electric motors, hydraulics, and pneumatics. For example, piezoelectric materials have been used as actuators to control wing panels $[14,15]$, spanwise deflection [16] and trailing-edge flaps [17,18]. As the actuation energy cannot be recovered, these actuators will cost energy in each cycle of operation, which will lead to a significant energy consumption in the operation lifetime. Therefore, some concepts have been proposed to reduce the required force or torque and therefore to allow smaller and lighter actuators, or even no actuator, to be employed to drive the system. For some typical cases, such as gravity equilibrators, a weight can be carried throughout its range of motion without any external energy by using spring mechanisms or 
counterweights [19-21]. Such static balancing is described as "zero stiffness" or "neutral stability", as the system can be moved without operating energy. Tensegrity structures [22] and shell structures [23] can also have zero-stiffness properties and thus large elastic deformation can be obtained without consuming external energy[24]. Furthermore, "quasi-zero-stiffness" mechanisms with negative stiffness elements have been extensively developed for vibration isolation [25-27].

In general, many systems have force that is purely a function of displacement, as shown in Fig. 1(a), known as an elastic force response. The traditional way to actuate a system with internal stiffness $k_{p}$ and the external load $L$, requires a high power actuator. In order to produce a static balancing for such system with a zero stiffness property, negative stiffness is required to assist the imposed deformation. Figure 1(b) shows a representative negative stiffness property, which has a completely opposite behaviour to the positive stiffness system and helps to construct an energy balancing system shown in Fig. 1(c). In other words, by coupling the negative stiffness mechanism, the total stiffness of the whole system becomes $k_{p}+k_{n}$ and the key benefit is that a lower power actuator can be used to generate the same output with regards to the same external load $L$.

As the displacement returns zero, the elastic energy stored in the driven structure will force the negative stiffness system back to its original state, with a net-zero actuation force. The negative stiffness mechanism can be coupled to the target positive stiffness system to produce an energy balancing system and the energy required to actuate the existing system can be balanced by the stored energy in the negative stiffness system. Therefore, Figure 1 shows that for the system assumed to be perfectly elastic, negative stiffness can drive the required actuation force to be zero. Although many real-world systems include significant inelastic forces, which can be rate or history dependent, or due to external disturbance, these cases represent a more complex problem that will be the subject of future work. In what follows, it is assumed that the driven structure has a purely elastic force-displacement response. It can be assumed that if the required input, such as force or torque, can be completely eliminated by the negative stiffness system, then in principle no energy is required to move the system, other than to overcome dissipation. Therefore, the use of negative stiffness systems is likely to benefit energy constrained systems, such as those present in the aerospace and automotive industries. Many 
applications have been proposed that use a negative stiffness for passive energy balancing. A negative stiffness nonlinear over-centre linkage is used on a tilt-rotor blade for active twist. This negative stiffness linkage mechanism uses the stored energy of a compressed spring to rotate the output shaft, resulting in an effectively softened blade that requires $70 \%$ reduction in torque for morphing [28]. In addition, a pulley based balancer has been proposed with a varying radius pulley to preserve moment equilibrium between a constant load and a varying spring length. By using such a spiral pulley negative stiffness mechanism, the required torque for the morphing actuation can be satisfactorily matched to create a nearly zero stiffness which required minimal energy [29,30]. A bidirectional torsional negative stiffness (BTNS) mechanism by using a series of pre-compressed springs has been investigated for energy balancing systems. This integrated BTNS mechanism was verified to tailor the kinematics of the required torque driving the active camber and the results showed a similar torque-rotation profile can be generated [31]. The proposed device provides actuation to change the state of the system, such as deforming a structure or lifting a mass; hence the energy provided by the actuator was transformed into an increased potential energy in the system. Once the system returns to its original state, all of the energy provided by the actuator will be recovered, if the system is conservative. Motion of the output occurs freely because energy can be transferred from the negative stiffness mechanism to the load, and back, with no change to the total energy in the system. However, the resulting passive energy balancing system would not necessarily be intrinsically stable, as the output position would be free to move significantly under small variations in the load, such as gusts. Therefore, it is intended that stability would be provided in any practical system through other features, such as non-back driveablility in the actuation system, for example using worm gearing and brakes. 


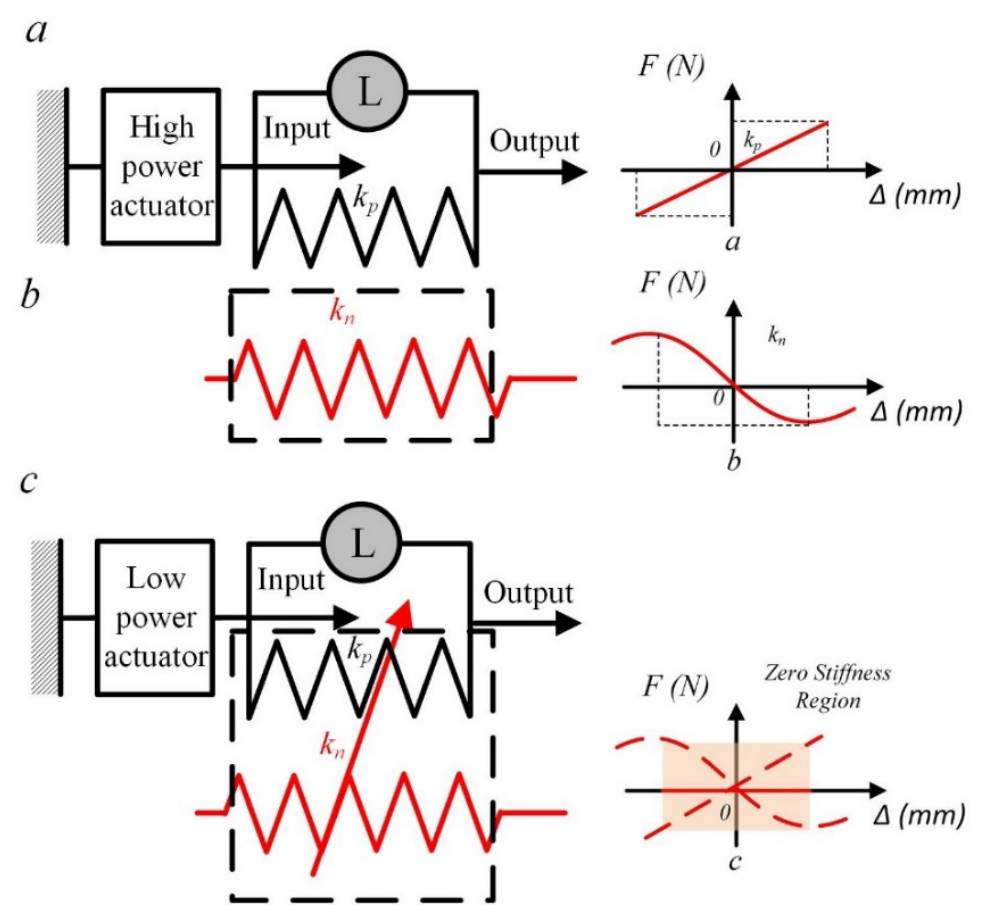

Figure 1. Minimalistic model of a negative stiffness mechanism for energy balancing. $L$ denotes the external load, $k_{p}$ is the internal stiffness of the load system and $k_{n}$ is the negative stiffness system. (a) Traditional actuated system. (b) The designed negative stiffness mechanism. (c) Energy balancing system by adding the negative stiffness mechanism

In the current study, a passive energy balancing mechanism designed for linear actuation is proposed. The mechanism is supposed to actuate the morphing wingtip, which is based on unsymmetrical stiffness of compliant structures $[15,16]$. An electrical linear actuator was integrated into the morphing wingtip, and has demonstrated the concept [17]. To satisfy the high actuation forces due to the structural stiffness and aerodynamic loads, the passive energy balancing mechanism is applied in the present paper. The proposed mechanism can provide linear actuation to deform the compliant structure and change the dihedral angle of the wingtip while minimising the external energy requirement. The kinematics of the torque shaft, the linear motion mechanism and rotation angle are investigated in Section 2. Then, the mechanism is used in the compliant structure of the morphing wingtip design in Section 3. A measurement method, which is based on the concept of coordinate transformation, is used to estimate the required actuation force of the compliant structure. A simplified model of this morphing wingtip structure [15-17] is fabricated with a passive energy balancing design and the corresponding required actuation force is compared to the morphing wingtip structure without the energy balancing system. 
Finally, an optimisation case is performed to further improve the performance of the passive energy balancing mechanism by exactly matching the requirements of the morphing actuation and the predicted required force.

\section{Negative stiffness mechanism for linear actuation}

The negative stiffness mechanism, which can provide a linear actuation force, is proposed in this section. The prototype of this energy balancing device is shown in Fig. 2.

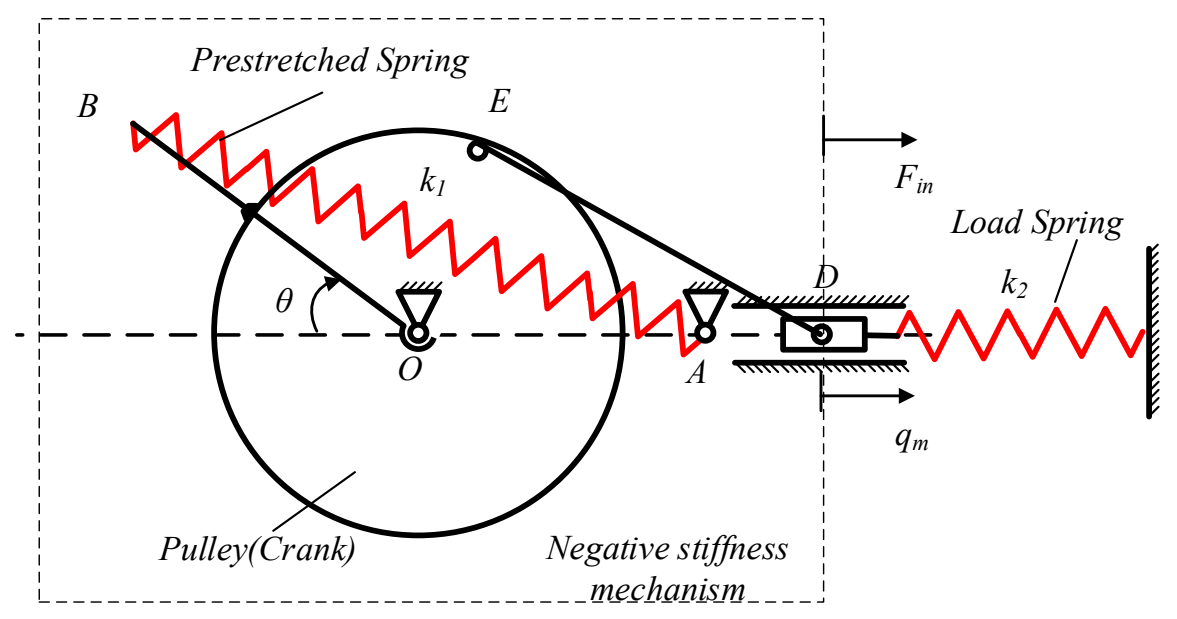

Figure 2. Schematic representation of a variable stiffness cam mechanism. In this mechanism $k$ denotes the stiffness of the linear spring, $q$ is the extra output position, $\theta$ is the input angle, while $q_{m}$ denotes the motion of the slider.

The torsional negative stiffness with an off-centre spring (TNSOCS) mechanism [32] is developed to produce the actuating force and the slider-crank mechanism is designed to transfer the torque to linear motion actuation. The shaft is centred at $O$ and $\theta$ is the shaft rotation angle. A prestretched spring is fixed on the shaft and point $B$ is the off-centre point for the spring. The slider-crank mechanism is connected to the torsional negative stiffness mechanism and point $E$ is the joint. Therefore, when the pulley rotates, the slider can move in the horizontal direction as annotated by $q_{m}$ and the load spring will be deformed.

In order to investigate the tailored nonlinear negative stiffness mechanisms for linear motion, the kinematics of the system can be derived as follows. Two springs are shown here, one is designed as an energy storage device for the negative stiffness system and another represents the load system. Therefore, the potential energy function of this mechanism is given by: 


$$
V\left(\theta, q_{m}\right)=\frac{1}{2} k_{1}\left(l-l_{0}\right)^{2}+\frac{1}{2} k_{2}\left(q_{m}\right)^{2}
$$

where $l_{0}$ is the initial length of the spring and $l$ is the length of the spring at its current position, which is a function of the rotation angle $\theta . k_{2}$ is the stiffness of the output system (i.e. morphing wingtip structure) and can be considered as a linear system when $k_{2}$ is a constant. Then, the additional required force imposed by the negative stiffness modulating mechanism is given by

$$
F_{i n}\left(\theta, q_{m}\right)=\frac{\partial V}{\partial q_{m}}=k_{1}\left(l-l_{0}\right) \frac{\partial l}{\partial q_{m}}+k_{2} q_{m}
$$

Moreover, Fig. 3 shows the slider-crank mechanism is a triangle and the displacement $q_{m}$ of the slider can be solved by using

$$
q_{m}=L\left(\left(\sqrt{1-\lambda^{2} \sin ^{2} \theta}-1\right)+\lambda(1-\cos \theta)\right)
$$

where $\lambda=R / L$ denotes the ratio of the radius of the crank shaft and the connecting rod and $q_{m}=0$ when $\theta=0$.

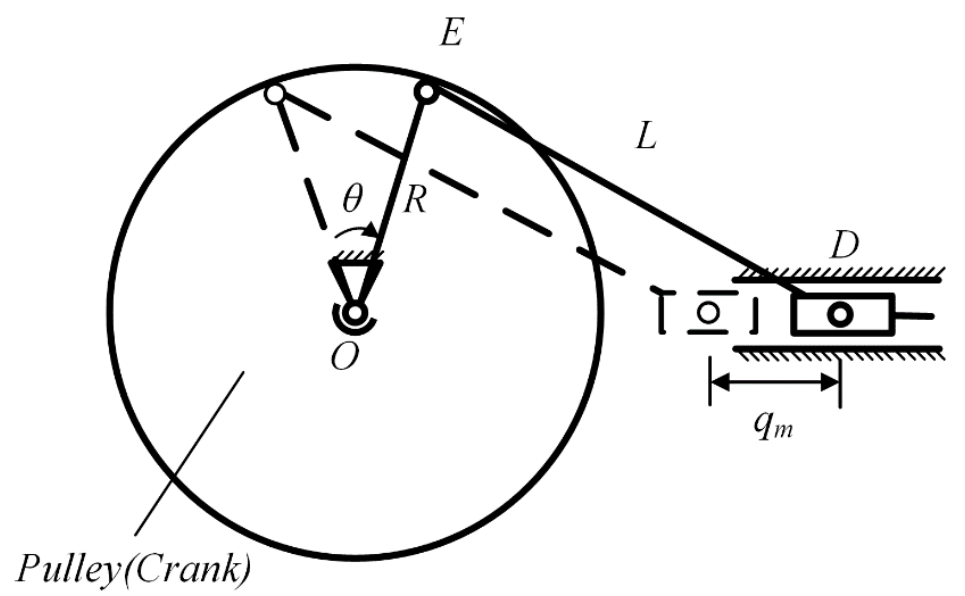

Figure 3. The slider-crank mechanism: displacement $q_{m}$ of the slider under different $\lambda$ and $\theta$.

Figure 4 shows the displacement of the slider under different $\lambda$ when the crank shaft rotates. It can be seen that if the value of $\lambda$ is increased, a larger displacement $q_{m}$, can be achieved. 


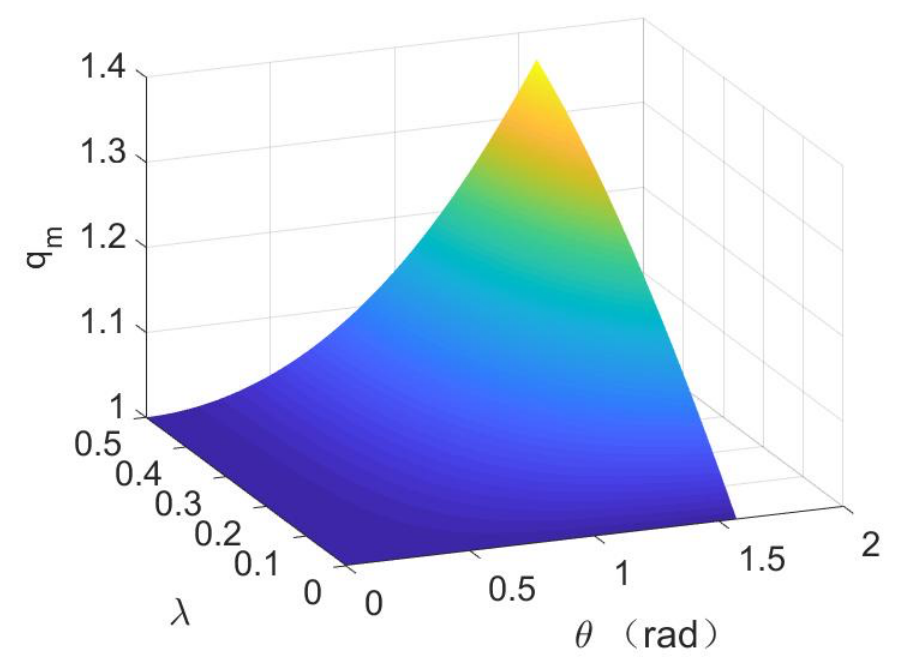

Figure 4. Displacement $q_{m}$ under different $\lambda$ and $\theta$.

Now, the kinematics of the torsional negative stiffness shaft are investigated. Figure 5 shows that the cable is fixed on the shaft and point $B$ is the off-centre point for the spring. The Cartesian coordinates can then be defined with the origin point at $A$ and the coordinates of any point $B^{\prime}$ can then be defined as

$$
\begin{gathered}
x_{B}=-l_{o f f}-b \cos \theta \\
y_{B}=b \sin \theta
\end{gathered}
$$

where $r$ is the length of the vector $\overline{O B}$ and $l_{\text {off }}$ is the length of the vector $\overline{O A}$.

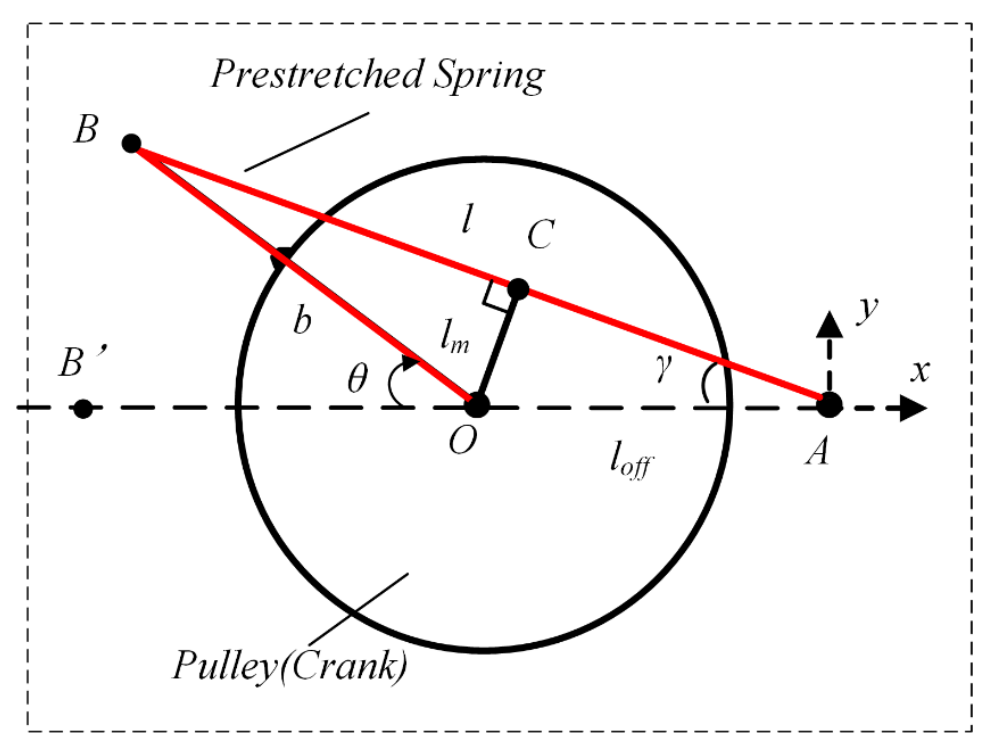

Figure 5. Torsional negative stiffness shaft geometry analysis with moment arm details. 
Therefore, the length of the vector $\overline{A B^{\prime}}$ is equal to

$$
l=\sqrt{x_{B}^{2}+y_{B}^{2}}=\sqrt{(b \sin \theta)^{2}+\left(l_{o f f}+b \cos \theta\right)^{2}}
$$

The change of the length $l$ along with shaft rotation angle $\theta$ can be calculated by

$$
\frac{\partial l}{\partial \theta}=-l_{o f f} b \sin \theta / \sqrt{(b \sin \theta)^{2}+\left(l_{o f f}+b \cos \theta\right)^{2}}
$$

The motion of the slider can also be calculated by

$$
\frac{\partial q_{m}}{\partial \theta}=L\left(\lambda \sin \theta-\lambda^{2} \sin \theta \cos \theta /\left(\sqrt{1-\lambda^{2} \sin ^{2} \theta}\right)\right)
$$

Therefore, the force can be obtained by substituting Eqs. (7) and (8) into Eq. (5) as

$$
F_{i n}\left(\theta, q_{m}\right)=\frac{\partial V}{\partial q_{m}}=k_{1}\left(l-l_{0}\right) \frac{\partial l}{\partial \theta} \frac{\partial \theta}{\partial q_{m}}+k_{2} q_{m}
$$

In Eq.(9), for an ideal energy balancing system, we require $F_{\text {in }}\left(\theta, q_{m}\right)=0$, where $k_{1}, b, l_{o f f}, L, l_{0}, \lambda$, $k_{2}$ are the system constants. For a potential actuation system with known $k_{2}$, and $q$, the parameters can be optimised for passive energy balancing. Moreover, Eq. (1) can be modified by employing $k_{1}=0$ to obtain the ordinary required moment about point $O$ to actuate the system without the proposed negative stiffness mechanism as

$$
\begin{gathered}
M_{r}=\frac{\partial V}{\partial \theta}=k_{2} L^{2}\left(\left(\sqrt{1-\lambda^{2} \sin ^{2} \theta}-1\right)+\lambda(1-\cos \theta)\right)(\lambda \sin \theta \\
\left.-\lambda^{2} \sin \theta \cos \theta /\left(\sqrt{1-\lambda^{2} \sin ^{2} \theta}\right)\right)
\end{gathered}
$$

Figure 6 shows the required moment and actuation force for the different input angles, it can be seen that the required moment is strongly nonlinear. This figure gives a schematic interpretation of the nonlinearity of such a mechanism, but the details such as rotation angle is based on the specific design. Moreover, the system will be turned from positive stiffness to negative stiffness after a specific angle. 


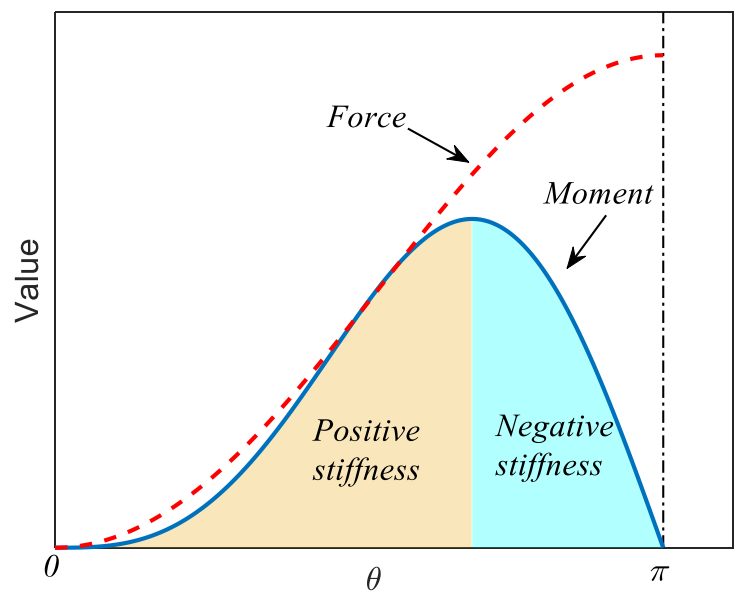

Figure 6. Required moment and actuation force as a function of input angle.

Therefore, in order to improve the performance of the total system, an initial established angle $\theta_{0}$ of the bar mechanism is proposed as an additional parameter as shown in Fig. 3. The displacement $q_{m}$ of the slider becomes

$$
\begin{array}{r}
q_{m}=L\left(\left(\sqrt{1-\lambda^{2} \sin ^{2}\left(\theta+\theta_{0}\right)}-1\right)+\lambda\left(1-\cos \left(\theta+\theta_{0}\right)\right)\right) \\
-L\left(\left(\sqrt{1-\lambda^{2} \sin ^{2}\left(\theta_{0}\right)}-1\right)+\lambda\left(1-\cos \left(\theta_{0}\right)\right)\right)
\end{array}
$$

where $\theta_{0}$ is the initial established angle. Moreover, the turning point $\theta_{t}$ of the system, which is the point of the maximum required moment in Fig.6 where the stiffness switches from positive to negative, can be obtained by setting

$$
\frac{\partial M_{r}}{\partial \theta}=0
$$

As the system is continuous with limited constants, it is hard to construct an energy balancing system that exactly cancels the moment for all $\theta$. Therefore, a metric is established to evaluate the performance of the energy balancing system. The energy conversion efficiency metric is evaluated theoretically from zero angle to the current position and is defined as

$$
\eta_{\text {eff }}=1-\int_{0}^{\theta}\left|\frac{F_{\text {in }} q_{m}}{M_{r} \theta}\right| d \theta
$$


This is a meaningful metric to evaluate the error for the passive energy balancing (PEB) system, as this implies the additional energy required during actuation. With this actuation force error metric, the geometric parameters of the system can be optimised using the fmincon function in Matlab as demonstrated in Section 3.5. The energy conversion efficiency metric $\eta_{\text {err }}$ is used as the objective function to make the torque available from the negative stiffness mechanism match as closely as possible the requirement to actuate the linear motion system over the entire prescribed rotation range.

\section{Passive energy balancing design for a morphing wingtip}

\subsection{Brief review of the morphing wingtip design}

A compliant structure, which was designed for the morphing wingtip, was proposed in earlier research [14]. The wingtip structure allows a variable dihedral angle whilst simultaneously supporting aerodynamic loads. The structure, as shown in Fig.7, consists of two corrugated panels with different stiffnesses, which are fixed at one end and connected by a relatively rigid part at the other end. The asymmetry of the two stiffnesses introduces axial-bending coupling to the structure, so that a linear actuator may be used to induce the required dihedral change. A morphing wingtip can consist of multiple units of such a compliant structure to obtain a larger induced deformation.
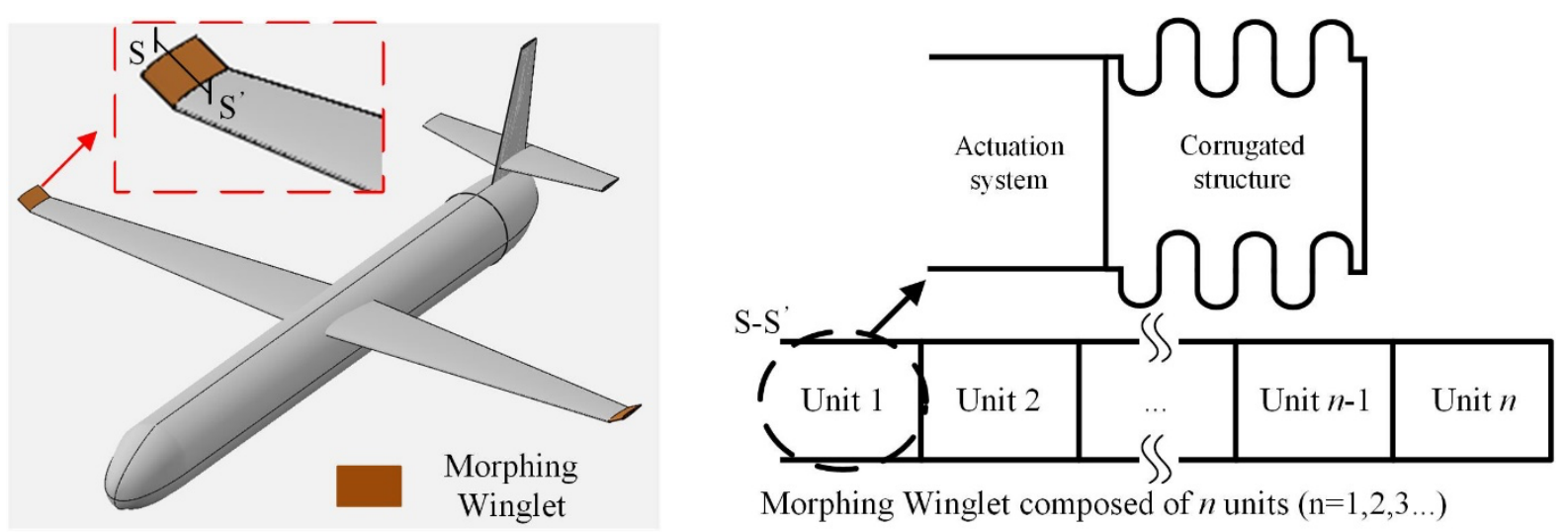

Figure 7. A conceptual design with the morphing wingtip array.

The deformation of the compliant structure was derived in earlier works [33-35]. The relationship between the deformation and the actuation force was derived in [14]. The axial and vertical deflections of the compliant structure are proportional to the magnitude of the actuation force. Optimisation of the structural stiffness was performed to reduce the required actuation force for a given deformation $[33,35]$. 
Since the shape-change comes from the elastic deformation of the compliant structure, the actuation force could still be very demanding even if the compliant structure was optimised, because the structure requires sufficient stiffness to withstand aerodynamic loads. Thus, the actuation system becomes critical, especially considering the constraints of the space within the aerofoil section, the weight and the output stroke. An electric linear actuator was adopted in the demonstrator of the proposed morphing wingtip design [35]. A medium order of deformation was achieved but even larger actuation forces would be necessary to achieve larger shape changes and higher flight speeds. Thus, the negative stiffness mechanism, which could provide a linear motion, with less energy consumption, is desirable.

\subsection{A coordinate transformation concept to estimate the actuation force}

To design the passive energy balancing device, the structural stiffness is required. However, the required actuation force of the compliant structure is often difficult to measure in a test machine as the induced deformation does not remain in the same direction. Although obtaining the deformation is straightforward when the structure is under a specific actuation force [35], it becomes more complicated to obtain the force as the induced deformation occurs in both the axial and vertical directions. Thus, a coordinate transformation is used in this section to estimate the actuation force.

Figure 8 gives a schematic representation of the coordinate transformation. Figure $8(a)$ shows the original boundary condition for actuating the proposed compliant structure, where one end of the structure is fixed and the linear actuator and the structure are pinned together. This connection can ensure that the linear actuation force can be provided and the rotation of the compliant structure cannot be affected by the actuator as shown in [33]. A measurement method based on the coordinate transformation concept shown in Fig. 8(b) is proposed to test the required actuation force of the compliant structure. The structure is pinned at both ends to allow the deformation in the $\mathrm{x}$ axis and the rotation of the side $B C$ around point $E$ so that the actuation force can be measured under the linear stretch. 


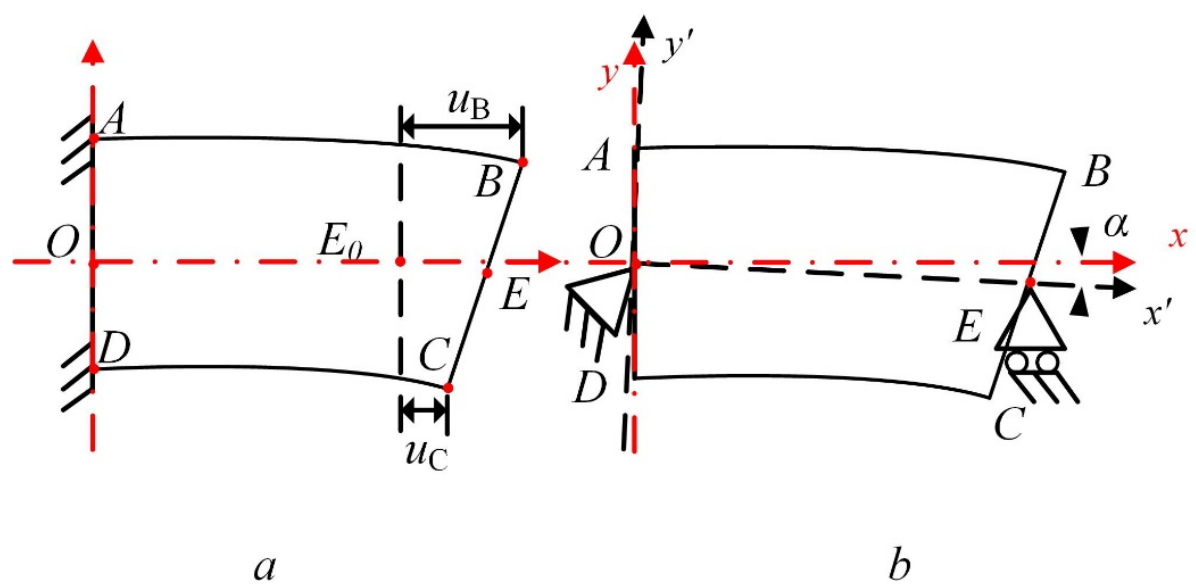

Figure 8. Schematic representation of the coordinate transformation for testing a compliant structure.

Two coordinate axes are shown here, axes $(x, y)$ are global coordinate and axes $\left(x^{\prime}, y^{\prime}\right)$ are attached to the structure, i.e. $x^{\prime}$ axis is defined to be parallel with centre line OE. Considering the actuation direction is always along the $x$ axis, a coordinate transformation can be used to rotate the coordinate axes $\left(x^{\prime}, y^{\prime}\right)$ in the counter-clockwise direction about the origin with the following coordinate transformation

$$
\left(\begin{array}{l}
x \\
y
\end{array}\right)=\left(\begin{array}{cc}
\cos \alpha & -\sin \alpha \\
\sin \alpha & \cos \alpha
\end{array}\right)\left(\begin{array}{l}
x^{\prime} \\
y^{\prime}
\end{array}\right)
$$

In the original coordinate system, the coordinate of point $E$ can be obtained as

$$
\left(\begin{array}{l}
x_{E} \\
y_{E}
\end{array}\right)=\left(\begin{array}{l}
x_{E}^{\prime} \cos \alpha-y_{E}^{\prime} \sin \alpha \\
x_{E}^{\prime} \sin \alpha+y_{E}^{\prime} \cos \alpha
\end{array}\right)
$$

in which $x_{E}^{\prime}=x_{E_{0}}+\Delta, x_{E_{0}} \gg \Delta$ and $y_{E}^{\prime}=0$.

The error between these two methods is

$$
\varepsilon=1-\frac{x_{E}}{x_{E_{0}}+\Delta}=1-\cos \alpha
$$

\subsection{Verification of the concept}

Because the induced rotation of the proposed compliant structure is moderate, the rotation can be small enough for error reduction, i.e. $\varepsilon \approx 0$. In order to verify the coordinate transformations concept, both of the two different boundary conditions are tested using finite element analysis. In both cases, the 
length, height and width of the compliant structure is $0.12 \mathrm{~m}, 0.1 \mathrm{~m}$ and $0.01 \mathrm{~m}$ respectively. The radius and length of the round corrugations remain $0.01 \mathrm{~m}$. The stiffness asymmetry is provided by introducing different thicknesses of the corrugated panels, which are denoted as $t_{1}$ and $t_{2}$ respectively. The finite element analysis is conducted in the commercial software Abaqus ${ }^{\circledR}$. S4R shell elements with a mesh size of $0.001 \mathrm{~m}$ are used in the detailed models [36]. The mesh size is sufficiently small to ensure convergence. Figure 9(a) shows the case when one end of the structure is fixed corresponding to Fig. 8 (a) and Fig. 9(b) shows the case when both ends are pinned corresponding to Fig. 8 (b).

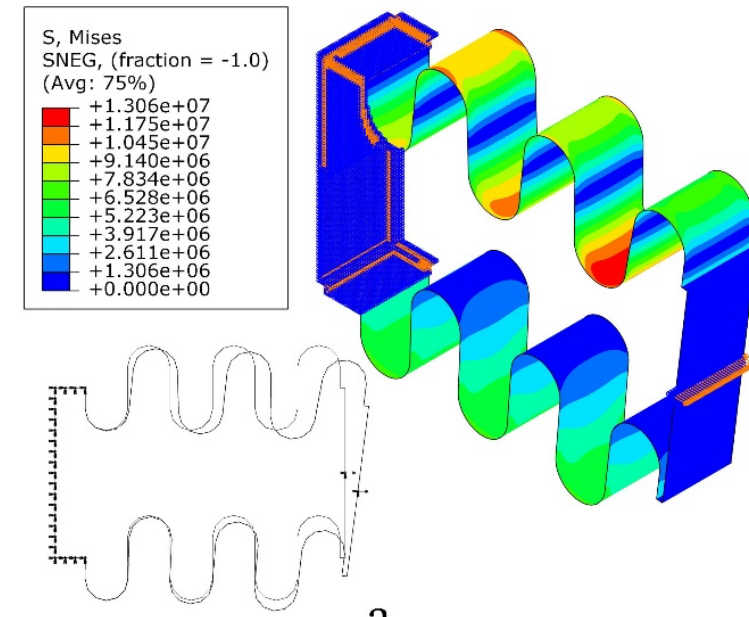

a

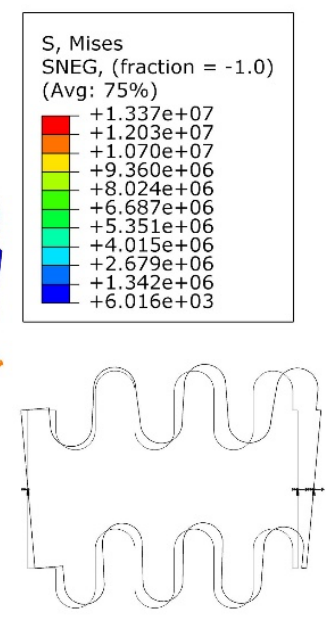

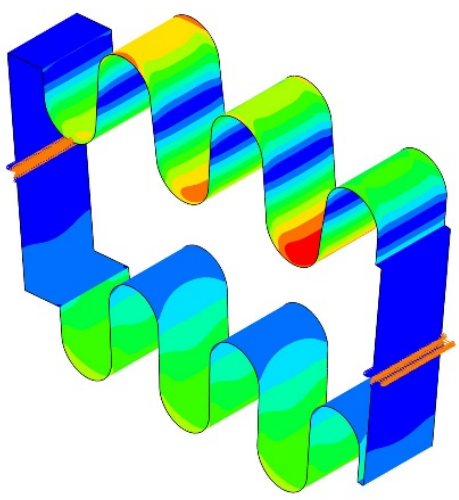

b

Figure 9. Finite element analysis results of the two different boundary conditions (unit of von Mises stress: Pa)

In the simulation, the actuation force is obtained when an increasing axial deflection (up to $0.01 \mathrm{~m}$ ) is applied to the structure. Different ratios of $t_{2} / t_{1}$ are simulated to show the results of different compliant structure models and Fig. 10 shows the results of final actuation force for a fixed horizontal deflection under the two proposed testing methods. It can be seen that the actuation force under the same vertical deflection is increased continuously when the stiffness asymmetry is increased (increased $t_{2} / t_{1}$ ) and the error between these two methods is very small.

Therefore, based on the theoretical analysis and the numerical simulation, the coordinate transformations concept can provide a simplified method to describe the deformation of the compliant structure and the required actuation with a reasonable accuracy. 


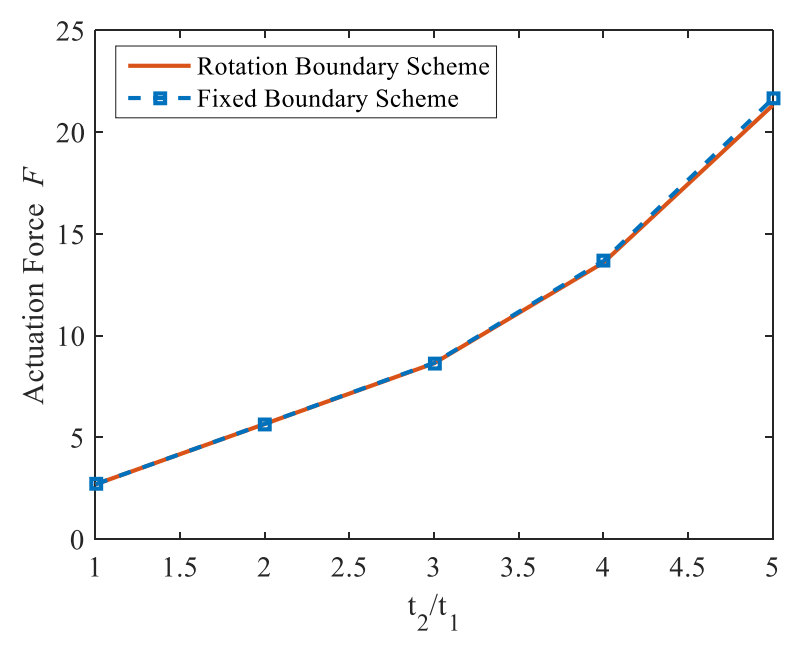

Figure 2. Verification of the compliant structure model based on different testing methods by detailed finite element analysis.

Following the theoretical analysis of the new testing method with pinned boundary conditions, a compliant structure is designed with $t_{2} / t_{1}=2$ and then manufactured using a $3 \mathrm{D}$ printer (Ultimaker 3D Printer model) for testing. A side view of the completed test device is shown in Fig.11, where two clamps are connected to the compliant structure to provide the pinned boundary conditions. A test machine (Zwick/Roell) is used to obtain the force-displacement curve. Additionally, round markers are stuck onto the surface of the structure to represent the shape change of the compliant structure as shown in Fig.11.

The experimentally measured force is now compared to the result from finite element analysis, which is shown in Fig.12. In this case, the maximum horizontal deflection is $0.01 \mathrm{~m}$ and the corresponding actuation force is $5.6 \mathrm{~N}$. The experimental curve is nearly linear and a minor offset of the force exists between the experimental result and the simulation prediction. In addition, the proposed compliant structure has a stiffness of approximately $560 \mathrm{~N} / \mathrm{m}$ and this value will be used for the passive energy design in the following Section. 


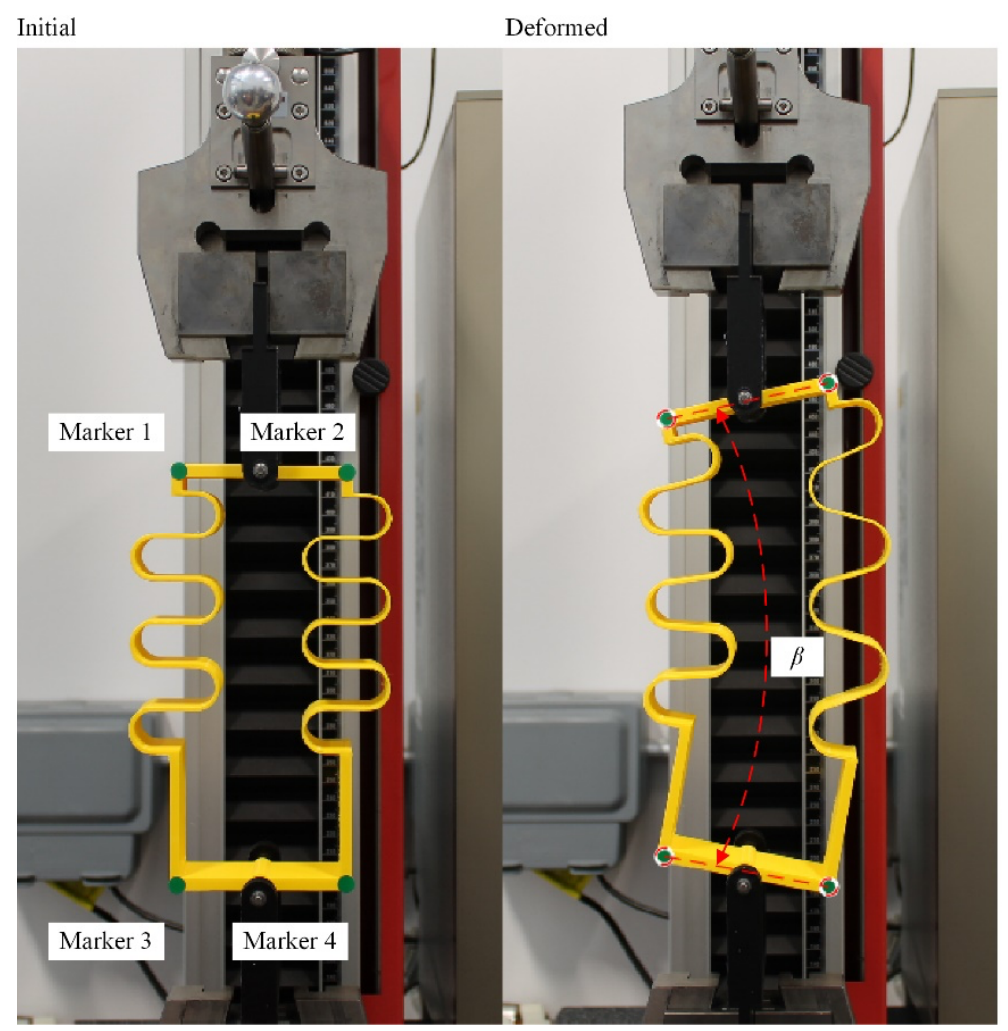

Figure 11. Deformation of the compliant structure in the test machine

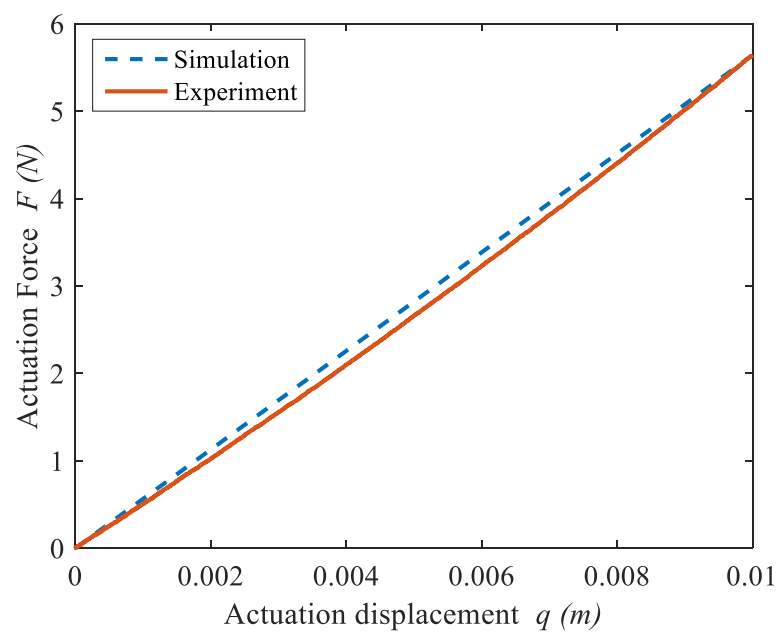

Figure 3. Actuation force and displacement for the compliant structure

\subsection{Negative stiffness design to reduce the actuation force}

In order to show the broad scope of applications envisioned for passive energy compliant structures, a generic design case is proposed here. The system is intrinsically scalable, limited mainly by the required components, such as drive springs and bearings. The test demonstrator is built to allow for experimental 
testing and validation of the passive energy balancing concept and the proposed analysis. Based on the investigation in Section 3.2, the stiffness of the proposed compliant structure $k_{2}=560 \mathrm{~N} / \mathrm{m}$ is used. The detailed parameters are shown in Table 1 and the corresponding initial rotation angle $\theta_{0}=167$ degrees, which is produced by shaft length $b$ and ratio $\lambda$. All the components used here are ubiquitous and already manufactured over a very broad range of scales.

Table 1. Parameters for passive energy balancing design

\begin{tabular}{c|cc}
\hline Parameter & Optimised value & Units \\
\hline Drive spring rate, $k_{1}$ & 0.069 & $\mathrm{~N} / \mathrm{mm}$ \\
Drive spring length, $l_{0}$ & 76.2 & $\mathrm{~mm}$ \\
Off-centre length, $l_{\text {off }}$ & 15 & $\mathrm{~mm}$ \\
Link bar length, $L$ & 74 & $\mathrm{~mm}$ \\
Shaft length, $b$ & 74 & $\mathrm{~mm}$ \\
Ratio $R / L, \lambda$ & 1 & - \\
Initial established angle, $\theta_{0}$ & 167 & degrees \\
\hline
\end{tabular}

Figure 13 shows the static test demonstration of a compliant structure with the negative stiffness design. The compliant structure is also 3D printed using the same printer and has an additional crossbar for mounting the link bar. The link bar and shaft were installed with the compliant structure and supported by bearings. Then, the spring is pre-tensioned and connected with the frame and rotation joint and a commercially available spring was sourced, resulting in an actual drive spring stiffness of $69 \mathrm{~N} / \mathrm{m}$ (Ashfield Spring Ltd; part number S.33).

Figure 14 shows the comparison between the theoretical and experimental results of the compliant structures with and without the negative stiffness design. The force provided by the negative stiffness mechanism shows a significant reduction when actuating the compliant structure. The maximum torque required by the extra actuator is nearly $0.6 \mathrm{~N} \cdot \mathrm{m}$, which is only $30 \%$ of the torque required by the compliant structure without negative mechanism, $1.5 \mathrm{~N} \cdot \mathrm{m}$. In this study, the negative stiffness mechanism provides a significant contribution to balance the positive stiffness system while providing a similar linear motion output. 

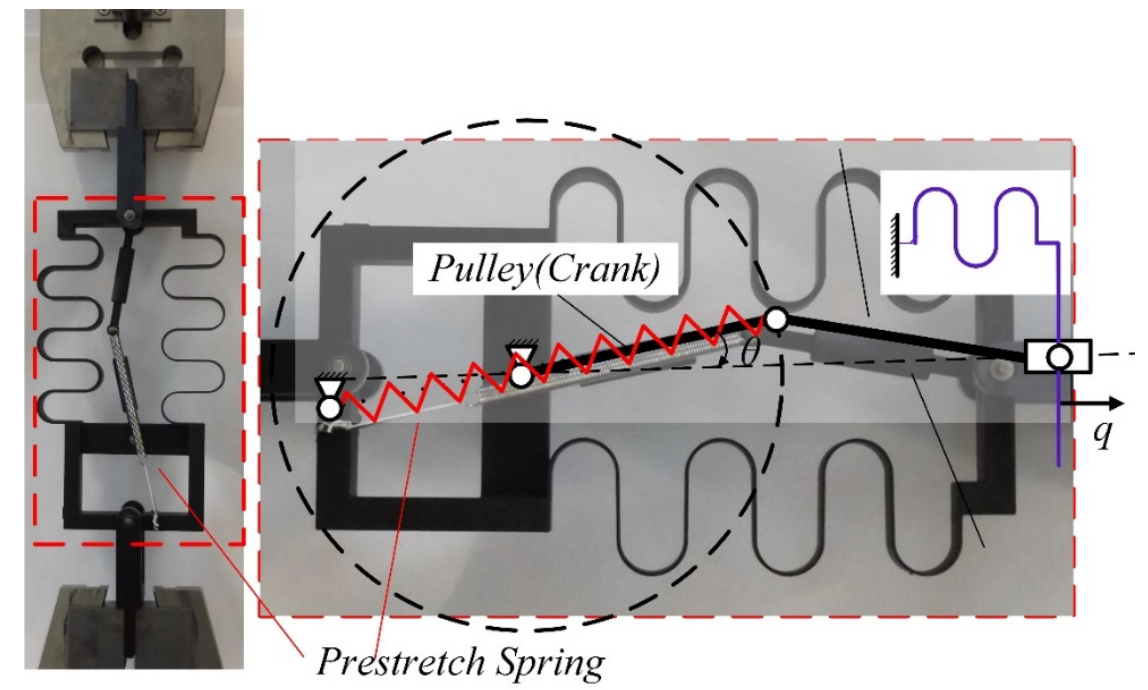

Figure 4. Static test demonstration with weak negative stiffness design.

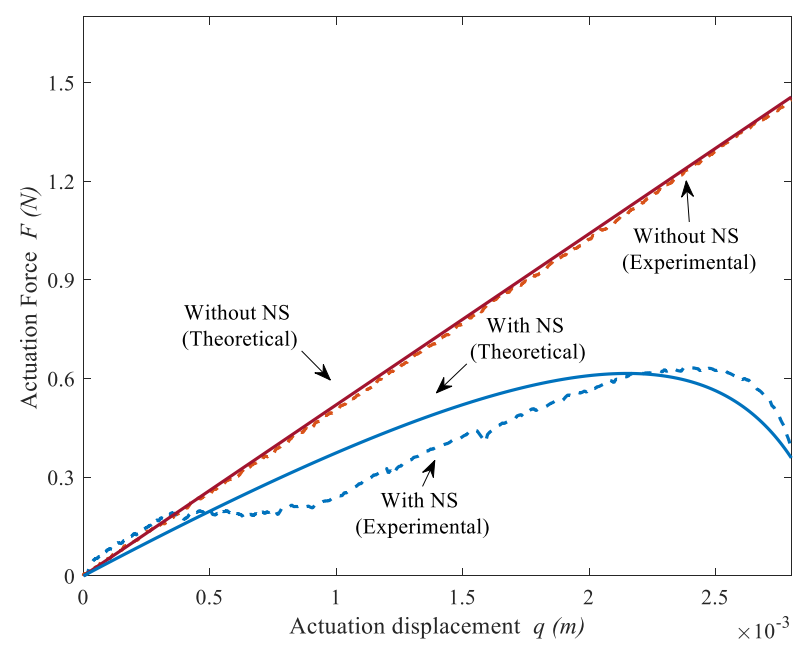

Figure 14. Comparison of the corrugated structure without and with negative stiffness design (dashed lines are experimental result and solid lines are theoretical results).

Figure 15 shows that the experimental result of required energy to actuate the compliant structure and the gross weight with and without the negative stiffness mechanism. With $20 \%$ additional gross weight, around $50 \%$ of the required actuation energy can be saved. If a smaller actuator can be used to provide the reduced actuation energy, the overall weight may still be reduced. And the overall performance of the morphing aircraft could be improved at the system level. Therefore, the concept shown here can be extended to many potential actuation design applications which need frequent state switching to reduce the mean power consumption and waste heat dissipation. High energy conversion efficiency can be provided by the pre-arranged extension spring and a smaller actuator can be used in the system. 


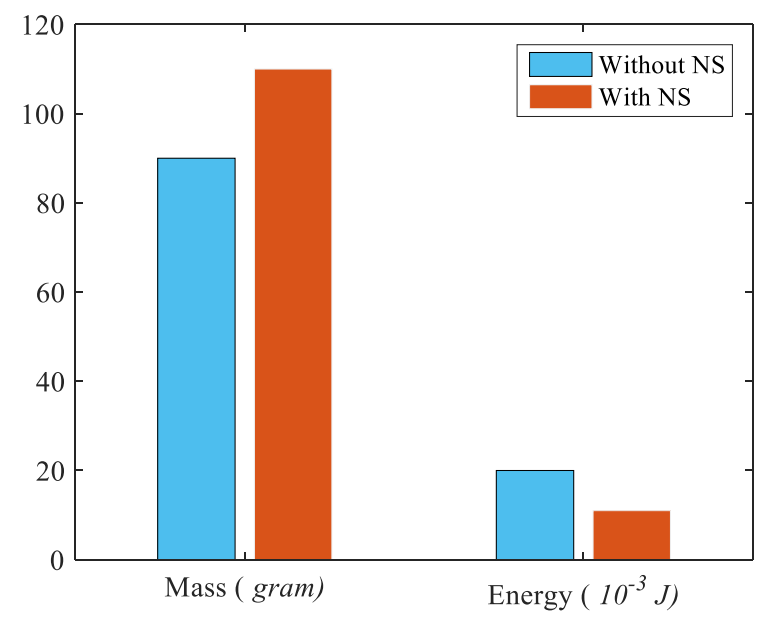

Figure 15. Comparison of the total mass and actuation energy.

Figure 16 shows a conceptual example of the passive energy balancing design for a compliant structure, used for the morphing wingtip. The structure consists of a compliant structure and a linear actuator is installed to deform the compliant structure. The negative stiffness mechanism is strategically located in parallel to the compliant structure.

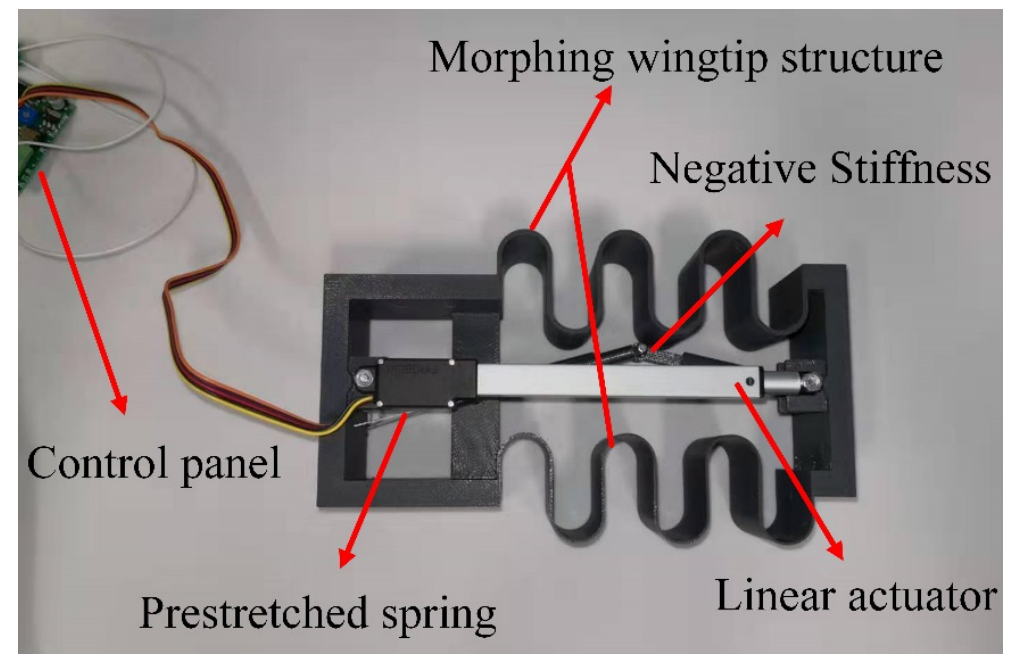

Figure 16. A compliant structure based on negative stiffness design.

\subsection{Optimisation for passive energy balancing}

In order to investigate how to use this negative stiffness mechanism for linear motion actuation, a linear spring stiffness $k_{2}=564 \mathrm{~N} / \mathrm{m}$ is chosen as the drive load for study and the rotation input angle is between 0 to $\theta_{t}-\theta_{0}$.

The objective is to maximise the energy conversion efficiency metric 
The nonlinear programmer solver, fmincon, in Matlab is used to optimise the parameters are shown in Table 2.

Table 2. Design parameters for the NS mechanism for linear motion optimisation.

\begin{tabular}{c|cccc}
\hline Parameter & Lower bound & Upper bound & Optimised value & Units \\
\hline Drive spring rate, $k_{1}$ & 0.01 & 1 & 0.11 & $\mathrm{~N} / \mathrm{mm}$ \\
Drive spring length, $l_{0}$ & 10 & 150 & 146 & $\mathrm{~mm}$ \\
Off-centre length, $l_{\text {off }}$ & 10 & 150 & 124 & $\mathrm{~mm}$ \\
Link bar length, $L$ & 10 & 150 & 130 & $\mathrm{~mm}$ \\
Shaft length, $b$ & 10 & 150 & 124 & $\mathrm{~mm}$ \\
Ratio $R / L, \lambda$ & 0.1 & 1 & 0.27 & - \\
Initial established angle, $\theta_{0}$ & 0 & 180 & 79 & degrees \\
\hline
\end{tabular}

With the optimised parameters obtained as discussed, and given in Table 2, the effectiveness of the optimal negative stiffness mechanism, which is used to construct an energy balancing system for the proposed target positive stiffness linear motion spring system, can be investigated. The performance predicted for the optimised torque shaft profile shows satisfactory matching of the linearised torque requirements. Figure 17 shows that the evolution of force with rotation for the spring and the linear motion system and the net torque of the whole system. The torque provided by the negative mechanism matches the torque required closely, and the maximum torque required by the extra actuator is nearly 0 $\mathrm{Nm}$.

Integrating the force against the displacement provides the mechanical energy required to actuate the compliant structure, as plotted in Fig. 18. By comparing these two cases, it shows that the negative stiffness mechanism has a strong ability to passively balance the required torque. Figure 18 shows that the predicted energy reduction is almost $98 \%$, with the energy required reduced from $0.85 \mathrm{~J}$ to $0.02 \mathrm{~J}$. This is due to the contribution of the energy stored in the extended spring in the initial position. 


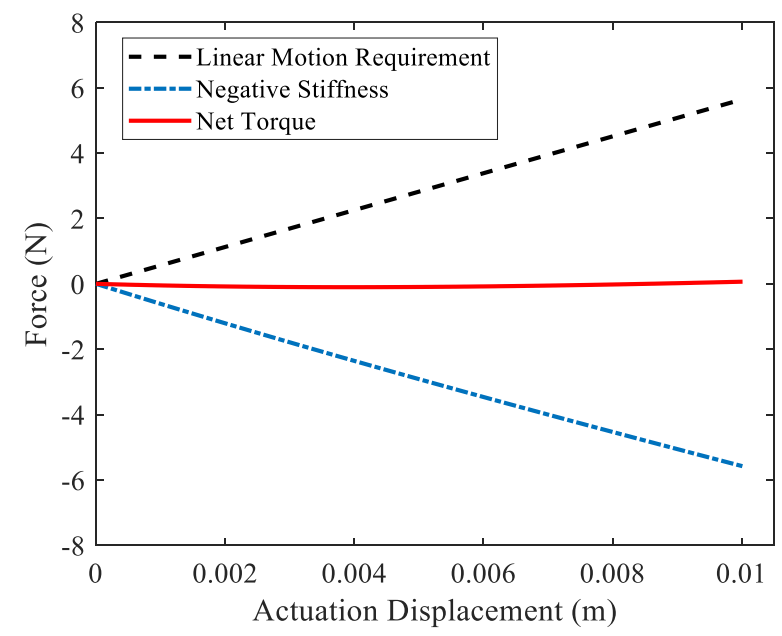

Figure 17. Predicted torque with optimised negative stiffness mechanism.

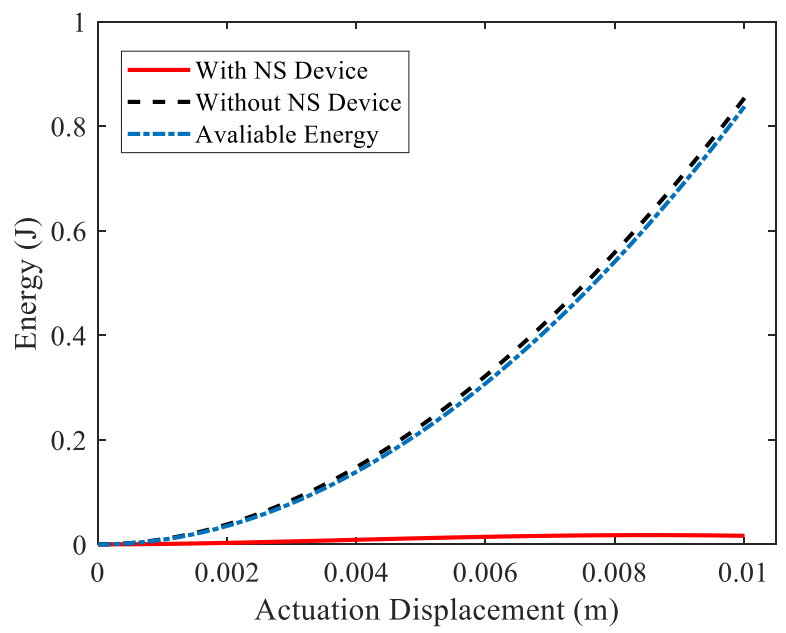

Figure 18. Comparison of predicted energy required with and without the passive energy balancing design.

Figure 19 shows the linear motion displacement along with the rotation angle provided by the negative stiffness mechanism. It can be seen that while there is a strong nonlinearity in the moment and rotation, the output displacement presents a linear relationship with the rotation during the optimised operation interval, which can also be seen in Fig. 6. Hence this characteristic implies that the negative stiffness mechanism can provide the required linear motion output by careful design and optimisation.

The energy conversion efficiency is shown in Fig. 20, which suggests that the optimised configuration of the negative stiffness mechanism provides significant benefits in terms of energy efficiency. 


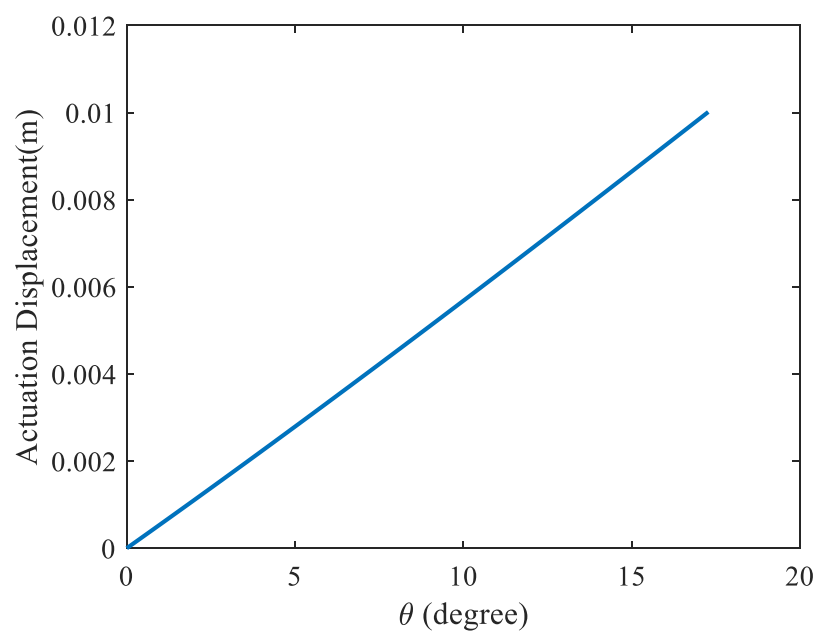

Figure 19. Predicted actuation displacement with rotation.

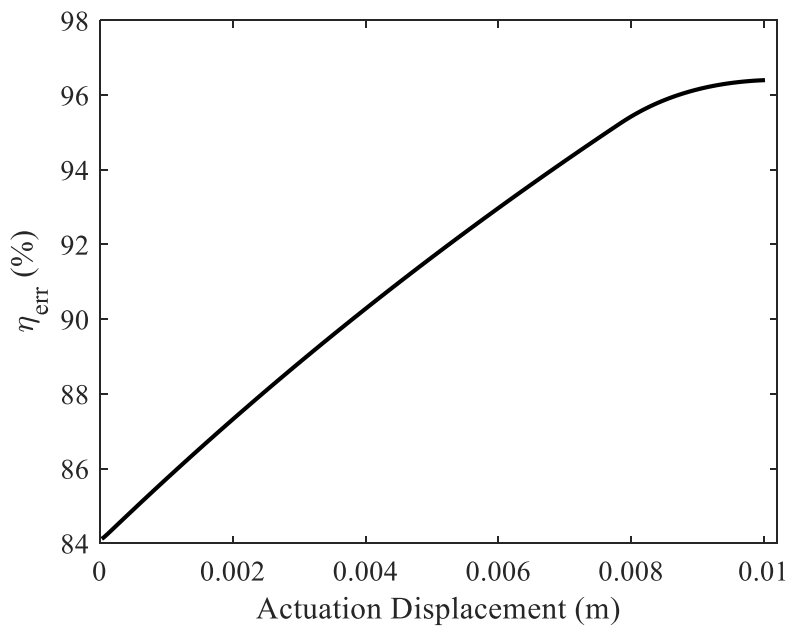

Figure 20. Evolution of efficiency with rotation.

\section{Conclusion}

A new conceptual design of the passive energy balancing system for a morphing wingtip structure has been presented. The design applies a torsional negative stiffness with an off-centre spring mechanism for linear motion actuation. The proposed negative stiffness device is designed to have the capability to passively balance the actuation requirement of systems with positive structural stiffness.

The motivation for using such a mechanism was presented and the kinematics of the tailored nonlinear negative stiffness mechanisms was introduced. A metric function was introduced to provide a basis for evaluation and also act as the objective function to optimise the geometry of the tailored nonlinear 
negative stiffness mechanism. The optimised negative stiffness mechanism was able to generate a torque that matches the required force of a linear spring closely, which means a significant contribution can be provided by the tailored nonlinear negative stiffness mechanism to convert the torque to linear actuation and balance the positive stiffness system. In addition, a testing method based on a coordinate transformation concept was applied to test the required actuation force of the compliant structure. The comparison between numerical simulation and experimental results was performed to verify the method. A case study, in which the passive energy balancing device was applied, showed a significant reduction of the force and energy requirements.

The proposed concept can be extended to other potential actuation design applications, which need frequent state switching to reduce the mean power consumption and waste heat dissipation. While the example used in this paper is relatively simplified, it provides an insight into the low energy actuation design problem, in which high actuation energy is required.

\section{Acknowledgement}

This research leading to these results has received funding from the European Commission under the European Union's Horizon 2020 Framework Programme 'Shape Adaptive Blades for Rotorcraft Efficiency' grant agreement 723491.

\section{References}

[1] A.K. Jha, J.N. Kudva, Morphing aircraft concepts, classifications, and challenges, in: Smart Struct. Mater. 2004 Ind. Commer. Appl. Smart Struct. Technol., 2004. https://doi.org/10.1117/12.544212.

[2] S. Barbarino, O. Bilgen, R.M. Ajaj, M.I. Friswell, D.J. Inman, A Review of Morphing Aircraft, J. Intell. Mater. Syst. Struct. 22 (2011) 823-877. https://doi.org/10.1177/1045389X11414084. 
[3] M. Arena, A. Concilio, R. Pecora, Aero-servo-elastic design of a morphing wing trailing edge system for enhanced cruise performance, Aerosp. Sci. Technol. (2019).

https://doi.org/10.1016/j.ast.2019.01.020.

[4] P. Panagiotou, K. Yakinthos, Aerodynamic efficiency and performance enhancement of fixedwing UAVs, Aerosp. Sci. Technol. (2019) 105575. https://doi.org/10.1016/j.ast.2019.105575.

[5] J.P. Eguea, G. Pereira Gouveia da Silva, F. Martini Catalano, Fuel efficiency improvement on a business jet using a camber morphing winglet concept, Aerosp. Sci. Technol. 96 (2020) 105542. https://doi.org/10.1016/j.ast.2019.105542.

[6] Z. Hui, Y. Zhang, G. Chen, Aerodynamic performance investigation on a morphing unmanned aerial vehicle with bio-inspired discrete wing structures, Aerosp. Sci. Technol. 95 (2019) 105419. https://doi.org/10.1016/j.ast.2019.105419.

[7] D. Xu, Z. Hui, Y. Liu, G. Chen, Morphing control of a new bionic morphing UAV with deep reinforcement learning, 2019. https://doi.org/10.1016/j.ast.2019.05.058.

[8] A. Gatto, F. Mattioni, M.I. Friswell, Experimental investigation of bistable winglets to enhance wing lift takeoff capability, J. Aircr. (2009). https://doi.org/10.2514/1.39614.

[9] S.W. Kim, J.Y. Lee, K.J. Cho, Towards a bistable morphing winglet for unmanned aerial vehicle(UAV), in: 2013 44th Int. Symp. Robot. ISR 2013, 2013. https://doi.org/10.1109/ISR.2013.6695648.

[10] A. Castrichini, V. Hodigere Siddaramaiah, D.E. Calderon, J.E. Cooper, T. Wilson, Y. Lemmens, Nonlinear folding wing tips for gust loads alleviation, in: J. Aircr., 2016. https://doi.org/10.2514/1.C033474.

[11] A. Castrichini, V.H. Siddaramaiah, D.E. Calderon, J.E. Cooper, T. Wilson, Y. Lemmens, Preliminary investigation of use of flexible folding wing tips for static and dynamic load alleviation, Aeronaut. J. (2017). https://doi.org/10.1017/aer.2016.108.

[12] R.C.M. Cheung, A. Castrichini, D. Rezgui, J.E. Cooper, T. Wilson, Testing of wing-tip spring device for gust loads alleviation, in: 58th AIAA/ASCE/AHS/ASC Struct. Struct. Dyn. Mater. Conf. 2017, 2017. https://doi.org/10.2514/6.2017-0630. 
[13] I. Dimino, G. Amendola, B. Di Giampaolo, G. Iannaccone, A. Lerro, Preliminary design of an actuation system for a morphing winglet, in: 2017 8th Int. Conf. Mech. Aerosp. Eng. ICMAE 2017, 2017: pp. 416-422. https://doi.org/10.1109/ICMAE.2017.8038683.

[14] R. Vos, R. Barrett, R. De Breuker, P. Tiso, Post-buckled precompressed elements: A new class of control actuators for morphing wing UAVs, Smart Mater. Struct. 16 (2007) 919-926. https://doi.org/10.1088/0964-1726/16/3/042.

[15] R. Vos, R. De Breuker, R.M. Barrett, P. Tiso, Morphing Wing Flight Control Via Postbuckled Precompressed Piezoelectric Actuators, J. Aircr. 44 (2007) 1060-1068. https://doi.org/10.2514/1.21292.

[16] S.A. Tawfik, D. Stefan Dancila, E. Armanios, Unsymmetric composite laminates morphing via piezoelectric actuators, Compos. Part A Appl. Sci. Manuf. 42 (2011) 748-756. https://doi.org/10.1016/j.compositesa.2011.03.001.

[17] T. Lee, I. Chopra, Design of piezostack-driven trailing-edge flap actuator for helicopter rotors, Smart Mater. Struct. 10 (2001) 15-24. https://doi.org/10.1088/0964-1726/10/1/302.

[18] S.R. Hall, E.F. Prechtl, Development of a piezoelectric servoflap for helicopter rotor control, Smart Mater. Struct. 5 (1996) 26-34. https://doi.org/10.1088/0964-1726/5/1/004.

[19] M.J. French, M.B. Widden, The spring-and-lever balancing mechanism, George Carwardine and the Anglepoise lamp, Proc. Inst. Mech. Eng. Part C J. Mech. Eng. Sci. 214 (2000) 501508. https://doi.org/10.1243/0954406001523137.

[20] R. Barents, M. Schenk, W.D. van Dorsser, B.M. Wisse, J.L. Herder, Spring-to-Spring Balancing as Energy-Free Adjustment Method in Gravity Equilibrators, in: Vol. 7 33rd Mech. Robot. Conf. Parts A B, 2009: pp. 689-700. https://doi.org/10.1115/DETC2009-86770.

[21] Y.-L. Chu, C.-H. Kuo, A Single-Degree-of-Freedom Self-Regulated Gravity Balancer for Adjustable Payload ${ }^{1}$, J. Mech. Robot. 9 (2017) 021006. https://doi.org/10.1115/1.4035561.

[22] M. Schenk, S.D. Guest, J.L. Herder, Zero stiffness tensegrity structures, Int. J. Solids Struct. (2007). https://doi.org/10.1016/j.ijsolstr.2007.02.041.

[23] S.D. Guest, E. Kebadze, S. Pellegrino, A zero-stiffness elastic shell structure, J. Mech. Mater. Struct. (2011). https://doi.org/10.2140/jomms.2011.6.203. 
[24] M. Schenk, S.D. Guest, On zero stiffness, Proc. Inst. Mech. Eng. Part C J. Mech. Eng. Sci. (2014). https://doi.org/10.1177/0954406213511903.

[25] P. Alabuzhev, A. Gritchin, L. Kim, G. Migirenko, V. Chon, P. Stepanov, Vibration Protecting and Measuring Systems with Quasi-Zero Stiffness, Hemisphere Publishing Co., Taylor \& Francis Group, New York, 1989.

[26] D.L. Platus, Negative-stiffness-mechanism vibration isolation systems, in: E.A. Derby, C.G. Gordon, D. Vukobratovich, P.R. Yoder, Jr., C.H. Zweben (Eds.), Proc. Vol. 3786, Optomech. Eng. Vib. Control, 1999: pp. 98-105. https://doi.org/10.1117/12.363841.

[27] A. Carrella, M.J. Brennan, T.P. Waters, Static analysis of a passive vibration isolator with quasi-zero-stiffness characteristic, J. Sound Vib. (2007). https://doi.org/10.1016/j.jsv.2006.10.011.

[28] D.J. Clingman, R.T. Ruggeri, Mechanical strain energy shuttle for aircraft morphing via wing twist or structural deformation, in: E.H. Anderson (Ed.), Proc. SPIE Smart Struct. Mater. 2004 Ind. Commer. Appl. Smart Struct. Technol., San Diego, CA, 2004: p. 288. https://doi.org/10.1117/12.538681.

[29] B.K. Woods, M.I. Friswell, Spiral pulley negative stiffness mechanism for passive energy balancing, J. Intell. Mater. Syst. Struct. 27 (2016) 1673-1686. https://doi.org/10.1177/1045389X15600904.

[30] J. Zhang, A.D. Shaw, A. Mohammadreza, M.I. Friswell, B.K.S. Woods, Spiral Pulley Negative Stiffness Mechanism for Morphing Aircraft Actuation, in: Vol. 5B 42nd Mech. Robot. Conf., ASME, 2018: p. V05BT07A003. https://doi.org/10.1115/DETC2018-85640.

[31] J. Zhang, A.D. Shaw, M. Amoozgar, M.I. Friswell, B.K.S. Woods, Bidirectional torsional negative stiffness mechanism for energy balancing systems, Mech. Mach. Theory. $131261-$ 277. https://doi.org/10.1016/j.mechmachtheory.2018.10.003.

[32] J. Zhang, A.D. Shaw, M. Amoozgar, M.I. Friswell, B. Woods, Torsional Negative Stiffness Mechanism for Bidirectional Morphing Aircraft Actuation, in: Proc. 6th Aircr. Struct. Des. Conf., Bristol, 2018. 
[33] C. Wang, H.H. Khodaparast, M.I. Friswell, A.D. Shaw, Compliant structures based on stiffness asymmetry, Aeronaut. J. 122 (2018) 442-461. https://doi.org/10.1017/aer.2017.144.

[34] C. Wang, H.H. Khodaparast, M.I. Friswell, Conceptual study of a morphing winglet based on unsymmetrical stiffness, Aerosp. Sci. Technol. 58 (2016) 546-558. https://doi.org/10.1016/j.ast.2016.09.015.

[35] C. Wang, H. Haddad Khodaparast, M.I. Friswell, A.D. Shaw, Y. Xia, P. Walters, Development of a morphing wingtip based on compliant structures, J. Intell. Mater. Syst. Struct. 29 (2018) 3293-3304. https://doi.org/10.1177/1045389X18783076.

[36] Dassault Systèmes, Abaqus 2016 Analysis User's Guide., 2015. 